\title{
La Segregación Invisible: Prácticas Punitivas y de Ordenamiento Académico en Escuelas Chilenas
}

\section{Invisible Segregation: Punitive Practices and Ability Grouping in Chilean Schools}

\author{
Verónica López ${ }^{1}$ * \\ Sebastián Ortiz ${ }^{1}$ \\ Claudio Allende ${ }^{2}$ \\ Juan Pablo Valenzuela ${ }^{2}$ \\ Luis González ${ }^{1}$ \\ ${ }^{1}$ Pontificia Universidad Católica de Valparaíso, Chile \\ ${ }^{2}$ Universidad de Chile, Chile
}

\begin{abstract}
Si bien las políticas educativas y la investigación en educación han abordado la segregación entre escuelas, las formas menos visibles de segregación y exclusión dentro de la escuela apenas se han estudiado. El objetivo de este estudio fue caracterizar y analizar la ocurrencia y concurrencia de dos tipos de prácticas: punitivas y de ordenamiento por habilidades. Se utilizó las bases de datos del sistema de información general de estudiantes de los años 2003 al 2018 para identificar prácticas de ordenamiento académico, y los cuestionarios que acompañan la prueba SIMCE del año 2018 para analizar las prácticas punitivas reportadas por estudiantes de $6^{\circ}(\mathrm{N}=224.274)$ y $10^{\circ}$ grado $(\mathrm{N}=202.282)$ y a padres de estudiantes de $4^{\circ}$ grado $(\mathrm{N}=214.211), 6^{\circ}$ grado $(\mathrm{N}=204.894)$ y $10^{\circ}$ grado $(\mathrm{N}=171.596)$. Los resultados muestran que los estudiantes de sexo masculino y en condición de pobreza reciben más prácticas punitivas, y que el ordenamiento académico es utilizado ampliamente en las escuelas chilenas, con mayor frecuencia en escuelas que solo imparten enseñanza secundaria. El uso de prácticas punitivas es más frecuente en aquellos colegios que ordenan académicamente. Se discute las implicancias de la sobrerepresentación de estas prácticas para los fenómenos de segregación educativa.
\end{abstract}

Descriptores: Segregación escolar; Agrupación escolar; Integración escolar; Exclusión social.

Even though educational policies and research in education have addressed betweenschool segregation, less visible forms of within-school segregation and exclusion have been scarcely studied. The purpose of this study was to characterize and analyze the occurrence and concurrence of two types of practices: academic ability grouping, and punitive disciplinary. The databases of the Chilean General Student Information System from 2003 to 2018 were used to identify ability grouping, and the questionnaires that accompany the 2018 SIMCE test was used to analyze punitive practices reported by students of 6 th $(\mathrm{N}=224,274)$ and 10th grade $(\mathrm{N}=202,282)$, and parents of 4 th grade students $(\mathrm{N}=214,211), 6$ th grade $(\mathrm{N}=204,894)$ and 10 th grade $(\mathrm{N}=171,596)$. Results show that male and poor students receive more punitive practices, and that ability grouping is widely used in Chilean schools, more frequently in schools that only provide secondary education. The use of punitive practices is more frequent in those schools that organize their classrooms according to academic ability. The challenges of over-representation in some students of these less visible practices of school segregation and exclusion in a school.

Keywords: School segregation; Academic grouping; School integration; Social exclusion.

*Contacto: veronica.lopez@pucv.cl

ISSN: $1696-4713$

www.rinace.net/reice/

revistas.uam.es/reice
Recibido: $\quad 27$ de abril 2020

$1^{\text {a }}$ Evaluación: 13 de julio 2020

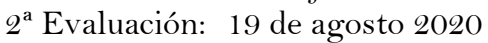

Aceptado: $\quad 7$ de septiembre 2020 


\section{Introducción}

No es novedad la preocupación de los gobiernos por incentivar reformas que apunten a combatir los problemas de segregación social y educativa de la educación (Perreza y Terigi, 2010). Estas reformas no sólo han maximizado las oportunidades educativas, garantizando el derecho a la educación y la inclusión de sus participantes (Cobeña, 2020), sino que han intentado hacer frente a las condiciones sociales y familiares del estudiantado, y que ampliamente predicen su posibilidad de éxito académico (Dupriez, 2010; Giménez et al., 2020; Murillo y Hernández, 2020; Sangsurin, Chusorn y Agsonsua, 2019)

En Chile, las estrategias implementadas para enfrentar la segregación son un caso paradigmático de estudio tanto en su estructura como organización (Allende, Valenzuela y Díaz, 2018; Duk y Murillo, 2020; Valenzuela, Bellei y De Los Ríos, 2014). Desde los años ochenta y durante la dictadura militar (1973-1990), se transformó en el único sistema del mundo en dónde se ha conferido a las familias total libertad de elección de escuelas para educar a sus hijos, primando una lógica de mercado asociado a sistemas de financiamiento a la demanda, políticas compensatorias para los más desventajados y sistemas de rendición de cuentas que garantizan la competencia interescuela (Parcerisa y Falabella, 2017; Valenzuela, Villaroel y Villalobos, 2013).

Actualmente este sistema coexiste con legislaciones de garantía de derecho e inclusión educativa, que eliminan el lucro y regulan los mecanismos de entrada a las escuelas públicas o municipales, y las instituciones particulares subvencionadas o semipúblicas con financiamiento estatal, pero con administración privada- $\left(\right.$ Ley $\left.\mathrm{N}^{\circ} 20.845,2015\right)$. Estas legislaciones buscarían atenuar los resultados en evaluaciones en rendimiento académico, que posiciona a Chile como uno de los países con mayores tasas de segregación entre escuelas (Bellei, 2013; OCDE, 2016; Valenzuela, Bellei y De Los Ríos, 2014), reportando incluso mayores niveles que los propios barrios y territorios de residencias de los estudiantes (Bellei, 2013; Elacqua y Santos, 2013), y que podría considerarse como un fenómeno de hipersegregación educativa (González, 2017).

En sistemas escolares altamente segregados con políticas recientes de inclusión en el acceso que buscan la recomposición del cuerpo estudiantil, como es el caso de Chile, es necesario prestar atención a nuevas formas de segregación (González, 2017). Chmielewski (2014) advierte que sistemas escolares que eliminan las barreras de entradas conducen a nuevos fenómenos de segregación intraescuela. Uno de ellos es el aumento en la frecuencia del uso de mecanismos de ordenamiento académico, que favorecerían fenómenos de discriminación positiva, así como uso de prácticas punitivas que favorecerían la recomposición del escenario escolar, con medidas de exclusión escolar. Estos fenómenos, para algunos autores, implicarían nuevas formas de segregación educativa, la cual estaría vinculada a una inclusión dentro de la exclusión: escuelas para ciertos sectores de la población, y experiencias escolares positivas sólo para los estudiantes más aventajados (Hopkins, 2003; Richard, Schneider y Mallet, 2012; Veiga-Neto y Corcini, 2012).

La evidencia en esta materia es preocupante. La literatura ha mostrado que la decisión de implementar mecanismos de ordenamiento interno sería una decisión de cada escuela, siendo en general un atributo del director del establecimiento (MINEDUC, 2009; Treviño et al., 2018). En Chile, este tipo de prácticas estarían bastante masificadas (Treviño et al., 2018), aun cuando no existe ningún tipo de regulación gubernamental que instruya o prescriba su uso. En Chile, según cifras de la OCDE (2013), aproximadamente un $74 \%$ del 
total de los colegios de educación secundaria declaró realizar agrupamiento por habilidad en todas $(34 \%)$ o algunas asignaturas $(40 \%)$, y solo cerca de un $26 \%$ no lo realizaría.

Este tipo de prácticas conviven con otras que la literatura ha denominado como punitiva (Skiba, Arredondo y Williams, 2014), definidas como sanciones disciplinarias contra estudiantes, y que explícitamente conlleva retirarlos temporal o prolongadamente de la instrucción académica, o recomponer los cursos de un mismo grado por problemas conductuales. Distintas investigaciones han mostrado que su uso desproporcionado es inefectivo para prevenir fenómenos de convivencia escolar (Skiba y Knesting, 2001) y tiene efectos directos en la segregación y exclusión social de las minorías (APA, 2008; Camacho y Krezmien, 2019; Skiba y Rausch, 2013).

En el sistema chileno, este tipo de prácticas han sido consideradas normales (López et al., 2011), y han contribuido con prácticas sutiles y cotidianas de segregación, o nuevos dispositivos de segregación (Bellei, 2013) que van "sacando" o "invitando a salir" del espacio escolar a estudiantes con comportamientos disruptivos (Ascorra et al., 2018; López et al., 2019; Muñoz et al., 2014; Trautmann, 2008). Asimismo, según los directores de los establecimientos chilenos participantes en la prueba PISA-2009, el 75\% de ellos declara su uso como causal de expulsión estudiantil; mientras que el 30\% lo utiliza como causa específica para la expulsión de los alumnos de bajo rendimiento (OECD, 2010). Asimismo, las prácticas punitivas de expulsión explicarían una parte significativa de la ventaja que obtienen los establecimientos de mejor rendimiento académico (Bellei, 2009).

A pesar de su importancia, no existen evidencias robustas que exploren este tipo de prácticas-punitiva y de ordenamientos- como nuevos mecanismos de segregación y exclusión educativa. Por el contrario, diversas investigaciones han reportado la necesidad de repensar la segregación educativa más allá de las legislaciones y las variables extraescolares, para pensar en cómo éstas se reproducirían en las prácticas cotidianas de las escuelas (Cobeña, 2020). De ahí que este estudio buscó explorar y caracterizar la ocurrencia de prácticas segregación/exclusión en el manejo de la diversidad estudiantil, profundizando el conocimiento sobre la segregación al interior de los establecimientos chilenos. Específicamente, este estudio aborda las prácticas de agrupamiento por habilidad entre cursos y su trayectoria a través del tiempo, y las prácticas punitivas que generan exclusión al interior de las escuelas.

\section{Prácticas de segregación por agrupamiento académico}

El debate académico sobre el agrupamiento por habilidad al interior de las escuelas como prácticas de segregación académica, se ha extendido por casi 100 años (Steengberger-Hu, Makel y Olszewski-Kubilius, 2016), sin existir hasta hoy consenso sobre sus efectos, a pesar de alta ocurrencia (OCDE, 2013) y diversidad de mecanismos de agrupamiento existentes (Dupriez, 2010).

En general, los mecanismos de agrupamiento por habilidad u ordenamiento académico implican acciones focalizadas para ordenar a los estudiantes entre clases (Ramberg, 2016). A pesar de la existencia de diferentes mecanismos (streaming o tracking, banding, setting, cross-age, entre otros), todos tienen como objetivo principal concentrar a estudiantes de 
similar capacidad académica, con el objetivo de promover prácticas pedagógicas pertinentes para cada grupo (Ireson y Hallam, 2001).

Por una parte, los promotores de estas políticas han indicado que la generación de cursos homogéneos facilita el trabajo de los docentes, permite una mayor especialización de las estrategias pedagógicas para alumnos con diferentes capacidades o habilidades, y contribuyen con una mayor equidad en el avance de los aprendizajes del conjunto de estudiantes de cada sala de clases (Bolick y Rogowsky, 2016; Duflo, Dupas y Kremer, 2011). En cambio, sus detractores han señalado que la aplicación de estos mecanismos podría perjudicar seriamente a los jóvenes que asisten a cursos con alumnos con menores habilidades, incrementando la inequidad, dañando el desempeño de corto y largo plazo, e influyendo en las expectativas, auto-estima, auto-concepto, motivación y confianza de los estudiantes (Belfi et al., 2012; Bolick y Rogowsky, 2016; Francis et al., 2017, 2019; Mazenod et al., 2018; Oakes, 2008; Tereshchenko et al., 2018).

Frente a esto, la literatura aún no es totalmente consistente en el reporte de los efectos observados ante políticas de ordenamiento académico. Por un lado, algunos estudios han mostrado que su aplicación maximizaría las oportunidades educativas para estudiantes con mayores capacidades, promueven su atención y participación durante la clase, y favorecen el desarrollo de actitudes positivas de los estudiantes rezagados sobre sí mismos y podría mejorar su rendimiento (Duflo, Dupas y Kremer, 2011; Figlio y Page, 2002). Por otro lado, otros estudios han mostrado que no existirían efectos sistemáticos asociados con el agrupamiento en distintas clases de estudiantes de alta, media o baja habilidad (Betts y Shkolnik, 2000; Dustmann, Puhani y Schönberg, 2017; Steengberger-Hu, Makel y Olszewski-Kubilius, 2016). En contraste, otras investigaciones, han concluido que los efectos académicos del agrupamiento son negativos, donde una de las causas probables sería que la mejora obtenida por los estudiantes aventajados no lograría compensar los efectos perjudiciales en los estudiantes de menor rendimiento (Dupriez, 2010; Ramberg, 2016), probando también efectos negativos en el desarrollo socioemocional del cuerpo estudiantil (Chmielewski, Dumont y Trautwein, 2013; Francis et al., 2017).

En Chile, la literatura de ordenamiento sobre gestión de la diversidad académica es muy incipiente y no existe información oficial sobre otros tipos de agrupaciones que operan al interior de los establecimientos escolares. En una primera investigación que buscó determinar la magnitud de este fenómeno, Treviño y coautores (2018), mostraron que cerca del $59 \%$ de los establecimientos que proporcionan solo educación media con dos o más cursos por grado, realizaría algún tipo de ordenamiento académico, y que estaría asociada con un aumento en tasas de deserción y repitencia de estudiantes. Así también, afectaría la posibilidad de conformar redes académicas y sociales entre estudiantes (Palacios et al., 2019), no así, con los niveles de autoestima y autoeficacia que mantendría efectos positivos los ambientes heterogéneos (Treviño et al., 2019).

Un resultado interesante de la evidencia empírica indica que, contrario a lo que se pudiera pensar, el agrupamiento interno en términos académicos y otros procesos de gestión de la heterogeneidad interna (como la expulsión) no son necesariamente contradictorios, siendo más bien procesos complementarios, que se presentaría a lo largo de todo el ciclo escolar (Treviño et al., 2016). Lo que sería crítico, en un escenario político y social que vuelve la inclusión de las diferencias un ideal necesario para la equidad de los sistemas escolares.

En relación a evidencia que haya explorado el manejo de la heterogeneidad más allá del ordenamiento académico y social, como una práctica de exclusión del estudiantado - 
concretamente, de cierto(s) tipo(s) de estudiante(s)- en el espacio escolar, Contreras y coautores (2020) muestran, por primera vez, evidencia que estudiantes con necesidades educativas especiales incorporados en escuelas que han implementado programas de integración escolar no serían agrupados entre clases al interior de dichas escuelas. Así mismo, muestran que a medida que mejores prácticas de inclusión son llevadas a cabo los efectos negativos derivados de incorporar estudiantes con este tipo de necesidades (en resultados) desaparecen (Contreras et al., 2020).

\section{Prácticas punitivas que generan exclusión}

En las últimas décadas, uno de los desafíos de la política de inclusión escolar ha sido promover acciones y estrategias que desarrollen una gestión transformativa y democrática de los ambientes escolares, en lugar de una gestión punitiva de control comportamental. Lo que en Chile ha resultado contradictorio y ambivalente (Magendzo et al., 2012; Morales, López, Ascorra y Carrasco, 2019), al coexistir estrategias políticas y legislaciones que por un lado, favorecen una aproximación formativa y preventiva en la gestión de conflictos mediante resoluciones pacífica, democrática y participativa; y por otro, una lógica punitiva que establece el control y el castigo como mecanismo para resolver los problemas convivenciales, y que tiene sus bases en las políticas de "Tolerancia Cero" implementadas en Estados Unidos hace más de dos décadas (Calvin, Gurel y Barber, 2017), y que hoy preocupan a la realidad latinoamericana (Lehmann et al., 2020; Morales y López, 2019) Esta segunda lógica supone un creciente uso de prácticas punitivas, definidas como sanciones contra los estudiantes que explícitamente conllevan retirarlos de la sala de clases por un tiempo significativo y que no necesariamente implican diálogo y reparación frente a los problemas escolares (Weaver y Swank, 2020), sino, más bien la exclusión del comportamiento disruptivo mediante la sanción (Campoy, 2019; Marcucci, 2019; Sugai et al., 2000), y en base a una diferenciación arbitraria de los agentes escolares sobre los estudiantes y sus condiciones de ingreso al espacio escolar (Lehmann et al., 2020; Marcucci y Elmesky, 2020; Williams et al., 2020). En Estados Unidos, se ha evidenciado que el nivel de ocurrencia o uso de este tipo de prácticas es más frecuente y está sobrerrepresentado en estudiantes de origen afroamericano (Bell, 2020; Camacho y Krezmien, 2019). En España, hay evidencia de una mayor ocurrencia en estudiantes con trastornos de apego (Campoy, 2019).

Distintas investigaciones han mostrado la ineficacia del uso de prácticas punitivas disciplinarias para prevenir la violencia (Skiba y Knesting, 2001; Skiba y Rausch, 2013; Weaver y Swank, 2020) impactando negativamente en las relaciones entre profesores y estudiantes y en la percepción de los estudiantes de la justicia educativa (APA, 2008; Peguero y Bracy, 2014; Augustine et al., 2018). Asimismo, tendrían implicancias en la segregación y exclusión social de las minorías (APA, 2008; Calvin, Gurel y Barber, 2017; Camacho y Krezmien, 2019; Luhmann et al., 2020; Marcucci y Elmesky, 2020; Peguero y Bracy, 2014; Skiba et al., 2014; Williams, et al., 2020), y en el fenómeno de la deserción escolar (Augustine et al., 2018; Peguero y Bracy, 2014; Skiba y Rausch, 2013).

En Chile, estudios cualitativos han mostrado que las prácticas punitivas de exclusión (suspensión, expulsión o traslado de los estudiantes a otro establecimiento) son frecuentes y de amplia aceptación en el quehacer cotidiano (López et al., 2011). Si bien las regulaciones vigentes, como la Circular $\mathrm{N}^{\circ} 1$ de la Superintendencia de Educación Escolar del 2014, se han orientado a tipificar este tipo de sanciones, siguen siendo aceptadas ya sea 
como justificación discursiva para comprender la convivencia escolar (Ascorra et al., 2018) o como mecanismos válidos para regular los diversos problemas de comportamiento al interior de las escuelas (López et al., 2011, 2019; Muñoz et al., 2014).

Ceardi, López y Valdez (2018) y Valdés, López y Chaparro (2018) examinaron los efectos de la percepción que los estudiantes tienen sobre la ocurrencia de prácticas punitivas de exclusión en la percepción de convivencia escolar inclusiva, encontrando no sólo una alta frecuencia en este tipo de prácticas, sino además correlaciones positivas con la percepción de prácticas de convivencia escolar inclusiva, democrática y pacífica. Asimismo, los resultados indicaron correlaciones positivas a nivel de escuelas entre las prácticas punitivas y las prácticas de convivencia escolar democrática, pacífica e inclusiva, lo que es sorprendente considerando que la literatura muestra que la existencia de prácticas de exclusión son asociadas con prácticas de segregación y un clima autoritario que socaba las posibilidades para el respeto, diálogo y participación en la escuela y para una convivencia más democrática e inclusiva (Augustine et al., 2018; Gregory et al., 2016; Losen, 2011; Umeh, Bumpus y Harris, 2019).

Sin embargo, no existe una caracterización numérica a gran escala de los distintos tipos de prácticas punitivas, ni muchos menos su vinculación con otros tipos de prácticas de segregación/exclusión, como las de ordenamiento académico.

\section{Método}

\subsection{Participantes}

Se utilizó las bases de datos del sistema de información general de estudiantes de Chile (SIGE) de los años 2003 al 2018. Estas consisten en un registro de características demográficas individuales y resultados académicos obtenidos por todos los estudiantes de Chile, y características descriptivas de los establecimientos educativos. Estos datos proporcionaron las variables promedio general y situación final al terminar el año en curso (aprobado, retirado o reprobado) utilizadas para determinar si un establecimiento realiza ordenamiento por habilidad.

Adicionalmente, se utilizó las bases de datos que corresponden a las pruebas del Sistema de Medición de Calidad de la Educación (SIMCE) del año 2018 aplicadas a todos los estudiantes en $4^{\circ}, 6^{\circ}$ y $10^{\circ}$ grado. Para este estudio se utilizó las encuestas complementarias aplicadas a los padres y apoderados, las cuales adicionalmente contienen información que permite caracterizar socio-demográficamente a los estudiantes del país, de $4^{\circ}$ grado $(\mathrm{N}=214.211), 6^{\circ}$ grado $(\mathrm{N}=204.894)$ y $10^{\circ}$ grado $(\mathrm{N}=171.596)$, y las encuestas a estudiantes de $6^{\circ}$ grado $(\mathrm{N}=224.274)$ y $10^{\circ}$ grado $(\mathrm{N}=202.282)$. En el año 2018 por primera vez se incluyeron preguntas que permiten identificar la magnitud de las prácticas punitivas en todo el país, diseñada por los autores y aceptadas para su incorporación en los cuestionarios previstos por la Agencia de Calidad de la Educación luego de una convocatoria abierta.

La muestra utilizada al año 2018 contó con 3.181 establecimientos con dos o más cursos (724 solo secundaria, 1036 solo primaria y 1421 completos) que corresponden a un $36 \%$ de todos los establecimientos (de primaria y secundaria) existentes en Chile. En términos de matrícula estos colegios corresponden aproximadamente a un $75 \%$ de la matricula total de niños y jóvenes en edad escolar. Con esto se asegura que los análisis realizados son representativos de una gran proporción del sistema educacional chileno, quedando 
subrepresentados principalmente los establecimientos de zonas rurales, que en general no tienen más de un curso por nivel.

\subsection{Instrumento}

Prácticas punitivas. Se incluyó un set de preguntas en cuestionarios de apoderados y estudiantes, para medir las percepciones de apoderados y estudiantes sobre la utilización de prácticas punitivas al interior de los establecimientos. Para el caso del cuestionario de apoderados incluyó siete preguntas con respuestas del tipo Likert, con rangos entre 1 a 5 $($ alfa $=0,82)$. El cuestionario de estudiantes incluyó 4 preguntas con respuestas del tipo Likert, con rangos entre 1 a 5 (alfa $=0,77$ ). Las preguntas utilizadas, sus escalas y la práctica punitiva que representan se muestran en el cuadro 1. Dado que el objetivo de esta investigación es determinar a modo general la existencia o percepción de ocurrencia de este tipo de prácticas al interior de los establecimientos se recodificaron los reactivos generando variables dicotómicas que toman el valor 1 , si una determinada práctica ocurrió una o más veces y o, si no ocurrió.

Cuadro 1. Preguntas sobre prácticas punitivas anuales y mensuales incluidas en SIMCE 2018 para padres/apoderados y estudiantes

\begin{tabular}{|c|c|c|}
\hline ACTOR & PREGUNTAS & $\begin{array}{l}\text { PRÁCTICA } \\
\text { PUNITIVA }\end{array}$ \\
\hline \multirow[t]{2}{*}{$\begin{array}{c}\text { Padres y } \\
\text { apoderados } \\
(\alpha=0,82)\end{array}$} & $\begin{array}{l}\text { Considerando el año pasado y este año ¿Le ocurrieron al } \\
\text { estudiante algunas de las siguientes situaciones? } 1: \text { nunca; } 2: 1 \\
\text { vez; 3:2 veces; 4:3 a } 5 \text { veces; 5:6 veces o más. } \\
\text { No lo dejaron participar de una actividad de la escuela } \\
\text { por mal comportamiento (por ejemplo, no ir a un } \\
\text { paseo de curso). } \\
\text { Lo dejaron condicional por mal comportamiento. } \\
\text { Lo suspendieron uno o más días por mal } \\
\text { comportamiento. } \\
\text { Lo cambiaron de curso por mal comportamiento. } \\
\text { Lo intentaron expulsar del establecimiento por mal } \\
\text { comportamiento. }\end{array}$ & $\begin{array}{l}\text { No } \\
\text { participación } \\
\text { Condicionalidad } \\
\text { Suspensión } \\
\text { Cambio de } \\
\text { curso } \\
\text { Intento de } \\
\text { expulsión }\end{array}$ \\
\hline & $\begin{array}{l}\text { En el último, ¿cuántas veces le ocurrieron al estudiante las } \\
\text { siguientes situaciones? } 1: \text { nunca; } 2: 1 \text { a } 2 \text { veces; } 3: 3 \text { a } 5 \text { veces; 4:6 a } \\
\text { 10 veces; } 5: 11 \text { veces o más. } \\
\text { Le pusieron anotaciones negativas en el libro de clases } \\
\text { por mal comportamiento. } \\
\text { Lo enviaron a inspectoría por mal comportamiento. }\end{array}$ & $\begin{array}{l}\text { Anotaciones } \\
\text { Negativas } \\
\text { Envío a } \\
\text { inspectoría. }\end{array}$ \\
\hline \multirow{2}{*}{$\begin{array}{c}\text { Estudiantes } \\
(\alpha=0,82)\end{array}$} & $\begin{array}{l}\text { En el último, ¿con qué frecuencia te ha ocurrido lo siguiente? } \\
\text { 1: nunca; } 2: 1 \text { a } 2 \text { veces; } 3: 3 \text { a } 5 \text { veces; } 4: 6 \text { a } 10 \text { veces; } 5: 11 \text { veces o } \\
\text { más. } \\
\text { Un profesor me puso anotaciones negativas en el libro } \\
\text { de clases por portarme mal. } \\
\text { Un profesor me envío a inspectoría por portarme mal. }\end{array}$ & $\begin{array}{l}\text { Anotaciones } \\
\text { Negativas } \\
\text { Envío a } \\
\text { inspectoría }\end{array}$ \\
\hline & $\begin{array}{l}\text { Considerando los dos últimos años ¿ंTe ocurrieron algunas } \\
\text { de las siguientes acciones? 1: nunca; 2: } 1 \text { vez; 3: } 2 \text { veces; } 4: 3 \text { a } 5 \\
\text { veces; 5: } 6 \text { veces o más. } \\
\text { Me suspendieron por portarme mal. } \\
\quad \text { Me cambiaron de curso por revoltoso. }\end{array}$ & $\begin{array}{l}\text { Suspensión } \\
\text { Cambio de } \\
\text { curso }\end{array}$ \\
\hline
\end{tabular}

Fuente: Elaboración propia.

Prácticas de ordenamiento académico. En Chile no existe ninguna política o decreto ministerial que exija a los establecimientos informar sobre los procesos de ordenamiento realizados, ni tampoco existe información pública que mencione si un establecimiento 
realiza este tipo de prácticas. Por ende, la identificación de los establecimientos que utilizan estas estrategias fue estimada de manera indirecta siguiendo las recomendaciones de Treviño y coautores (2018). Así, se identificaron tres mecanismos de agrupamiento por habilidad al interior de los colegios chilenos: a) agrupamiento inicial por habilidad, consiste en ordenar a los alumnos cuando ingresan al primer grado donde un establecimiento educacional provee educación, generando una distribución no aleatoria de sus estudiantes; b) reordenamiento académico, consiste en reasignar a más del $20 \%$ de los estudiantes, motivado por criterios académicos, de un mismo nivel de un año a otro, uno o más años desde su ingreso al colegio y; c) agrupamiento académico, consiste en la distribución intencionada de estudiantes nuevos y/o repitentes en niveles no iniciales.

La identificación de estos tipos de agrupamiento se realizó mediante un análisis de la distribución del rendimiento académico, previo al año analizado, de los estudiantes. Para ello se utilizaron tres variables: la proporción de estudiantes repitentes, y los alumnos que se ubicaban en el primer y quinto quintil del promedio de notas general obtenido el año inmediatamente anterior al analizado. Para identificar la existencia de alguno de los mecanismos mencionados se utilizó el test Chi-Cuadrado, para estimar la significancia en la distribución entre las aulas que poseen los establecimientos. En caso de haber diferencias significativas, se puede concluir que existiría una alta probabilidad que presente una distribución no aleatoria de estudiantes en el nivel evaluado. Luego se clasificó cada año y tipo de agrupamiento por habilidad a través de una variable dicotómica que tomó el valor 1 si dicho establecimiento utilizó dicho mecanismo en al menos un grado y o si no.

Finalmente, se clasificó cada establecimiento en cada tipo de agrupamiento definido considerando la persistencia de la(s) práctica(s) de agrupamiento en el tiempo, determinando (a) No hace ordenamiento, si a lo más se estimó que había agrupado un año; (b) Hace ordenamiento, si agrupó a sus estudiantes cuatro o más años y obligatoriamente lo hizo el último año observado y; (c) Hace ordenamiento esporádicamente (dos casos), si hace agrupamiento entre dos o tres años, y si agrupa estudiantes cuatro o más años, sin haberlo realizado durante el último año observado. Con este algoritmo fue posible clasificar a todos los establecimientos con 2 o más cursos en una de las categorías antes mencionadas desde los años 2007 al 2018 inclusive.

\section{Resultados}

\subsection{Ocurrencia de prácticas de ordenamiento académico}

Las figuras 1 y 2 muestran los resultados obtenidos para los tres tipos de ordenamiento académico analizados, diferenciando por tipo de enseñanza (solo primaria, solo secundaria y completos). Como se observa, las prácticas de ordenamiento siguen siendo utilizadas por la gran mayoría de los establecimientos tanto en primaria como en secundaria (Treviño et al., 2018). Todas las prácticas de agrupamiento por habilidad tienen una persistencia más alta en establecimientos que proveen solo educación secundaria, las que, si bien se han reducido significativamente en el periodo, siguen siendo utilizadas por su mayoría. Así mismo, la práctica de agrupamiento académico es el mecanismo más utilizado (ya sea recurrente o esporádicamente) por todos los colegios independiente del nivel al que proveen educación. 
Entre 2007 y 2018 ha disminuido la cantidad de escuelas que realizan prácticas de ordenamientos, sin observarse un aumento de casos que utilizan esporádicamente estas prácticas. Específicamente, las prácticas de agrupamiento inicial y reordenamiento académico presentaron la caída más pronunciada, siendo el agrupamiento inicial aquella que registró una mayor caída, de casi 25 puntos porcentuales en el transcurso de 10 años. Llama la atención la caída observada en el periodo 2014-2015 y posteriores del uso de estas prácticas de ordenamiento al interior de las escuelas.

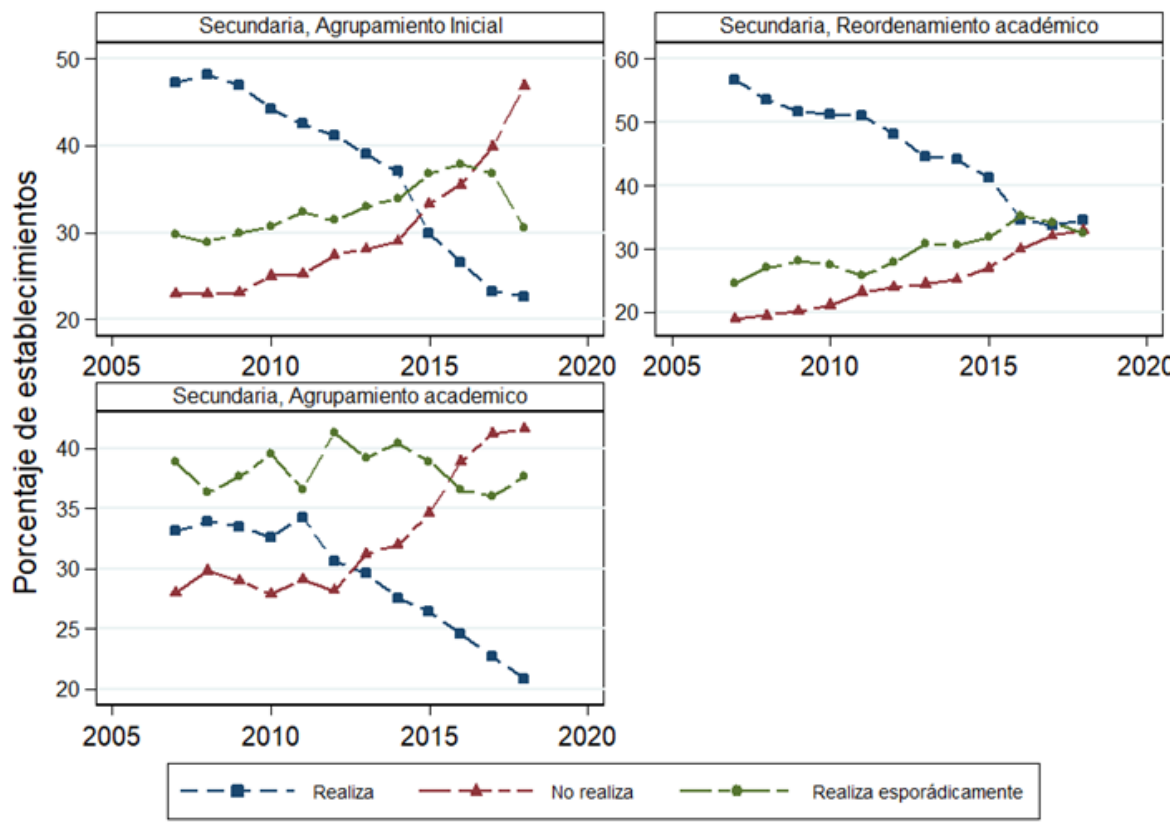

Figura 1. Evolución de la magnitud de uso de los tipos de ordenamiento identificados en establecimientos que proveen solo educación secundaria entre 2007 y 2018

Fuente: Elaboración propia.

La figura 1 muestra los resultados para los establecimientos que proveen solo educación primaria y colegios completos (primaria y secundaria). El reordenamiento académico es la práctica menos utilizada por este tipo de colegios, donde en establecimientos que proveen solo educación primaria prácticamente no es utilizada al año 2018. Sin embargo, tal como fue mencionado, el agrupamiento académico es la práctica más recurrente en estos establecimientos y sería aplicada principalmente de forma esporádica.

\subsection{Ocurrencia de prácticas punitivas}

En el cuadro 2 se observa las magnitudes de ocurrencia percibidas de las distintas prácticas punitivas, tanto por padres como por alumnos. En todos los niveles encuestados, las prácticas punitivas más comunes fueron las suspensiones y la condicionalidad, cuyo uso adicionalmente aumenta en grados más altos. Los reportes de suspensiones por parte de los estudiantes son mayores que las de los padres, situación que podría estar reflejando la existencia de otros tipos de suspensiones que los padres no tienen conocimiento. El cambio de curso sería la práctica de menor persistencia según el reporte de padres no superando el 1,3\%, situación que para los estudiantes es más común $(6 \%$ y 3,3\%, en 6 y 10 grado, respectivamente).

Finalmente, la no participación en actividades pareciera ser una práctica utilizada regularmente y con mayor intensidad en primaria, que en secundaria. En secundaria se 
optaría principalmente por prácticas que implican castigos mucho más severos como son las suspensiones y la condicionalidad de los estudiantes.

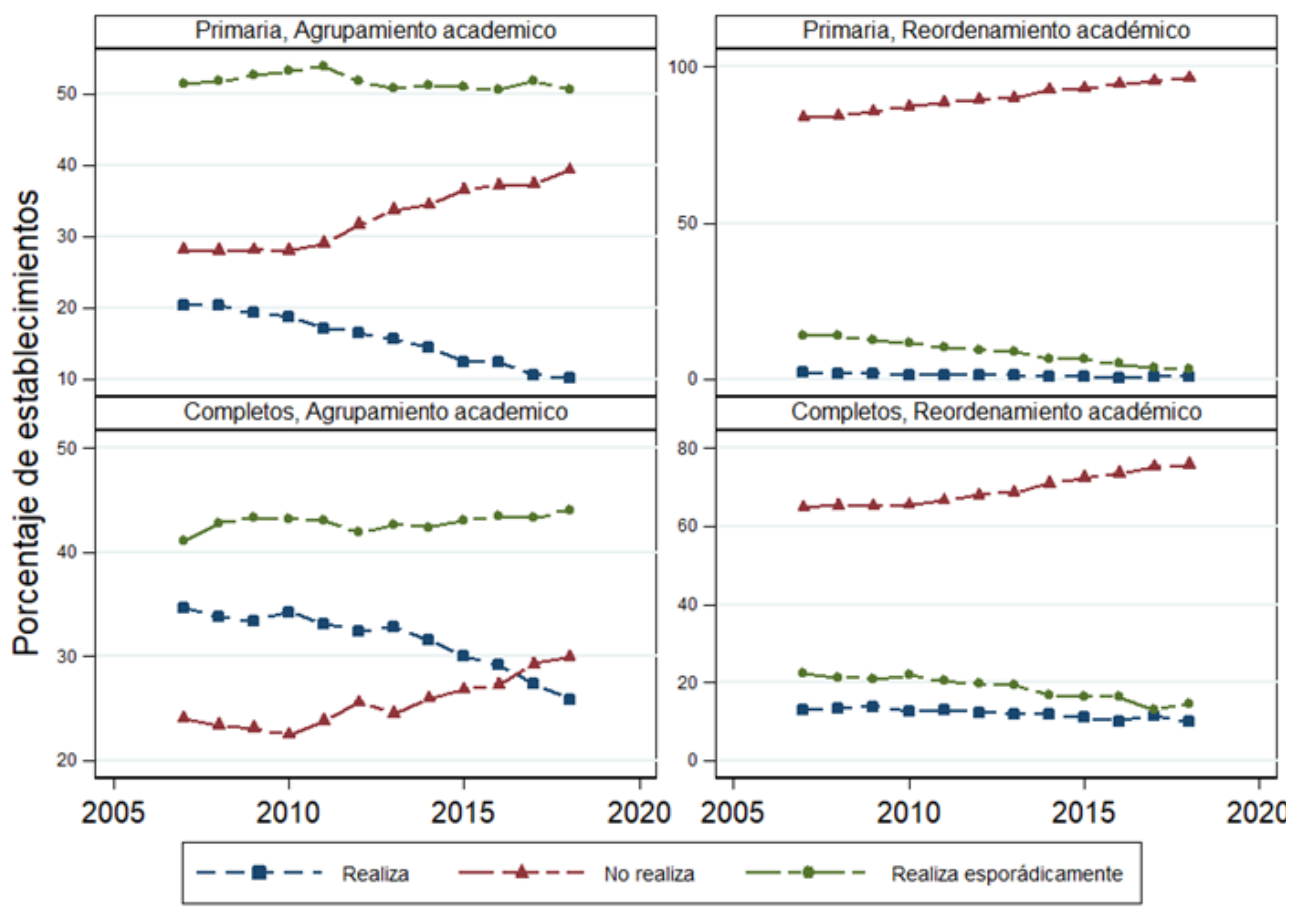

Figura 2. Evolución de la magnitud de uso de los tipos de ordenamiento identificados en establecimientos que proveen educación primaria y completos entre 2007 y 2018

Fuente: Elaboración propia.

Cuadro 2. Porcentaje percibido por padres y estudiantes de aplicación de prácticas punitivas en los últimos dos años (y número de padres/estudiantes que contestaron la pregunta)

\begin{tabular}{lccc}
\hline \multirow{2}{*}{ Práctica PUNitiva } & \multicolumn{3}{c}{ CURSO } \\
\cline { 2 - 4 } & $\mathbf{4}^{\circ}$ grado & $\mathbf{6}^{\circ}$ grado & $\mathbf{1 0}^{\circ}$ grado \\
\hline Percepción de Padres & & & \\
No Participación de actividades & $5,30 \%(207.055)$ & $6,20 \%(196.953)$ & $4,10 \%(164.438)$ \\
Condicionalidad & $4,60 \%(204.987)$ & $6,80 \%(195.226)$ & $7,80 \%(163.745)$ \\
Suspensión & $6,90 \%(204.609)$ & $9,90 \%(195.050)$ & $10,00 \%(163.661)$ \\
Cambio de curso & $1,10 \%(204.863)$ & $1,30 \%(195.195)$ & $1,30 \%(163.804)$ \\
Intento de expulsión & $1,70 \%(204.962)$ & $2,40 \%(195.298)$ & $2,20 \%(163.892)$ \\
\hline Percepción de Estudiantes & & & \\
Suspensión & & $15,10 \%(215.989)$ & $15,70 \%(199.046)$ \\
Cambio de curso & & $6,00 \%(214.098)$ & $3,30 \%(198.640)$ \\
\hline
\end{tabular}

Nota: En paréntesis el total de observaciones para cada pregunta de los cuestionarios respectivos. Fuente: Elaboración propia.

La figura 3 muestra las diferencias observadas en los reportes de prácticas punitivas, diferenciando por sexo y el nivel socioeconómico (NSE) de procedencia según quintiles de ingreso de las familias. Se observa de forma consistente una gran diferencia entre hombres y mujeres en todos los grados considerados, con mayor aplicación en hombres. Las diferencias fueron significativas para todas las prácticas punitivas consideradas (tanto 
padres como estudiantes) y niveles. Esta diferencia se atenuaría en el paso de educación primaria a secundaria, sin embargo, continúan siendo significativa.

Las diferencias antes descritas se incrementarían al considerar el NSE de procedencia de los estudiantes. Así, la aplicación de prácticas punitivas pareciera ser incremental a menores ingresos. Además, todas las prácticas punitivas ocurrirían con mayor frecuencia en establecimientos donde asisten estudiantes más vulnerables (quintil 1). Se encuentran diferencias significativas respecto a la frecuencia de aplicación en todas las prácticas punitivas aplicadas a sus pares pertenecientes al NSE más alto (quintil 5). Estas diferencias se exacerban aún más si consideramos las diferencias por sexo, llegando a niveles muy altos en algunas prácticas como las suspensiones, las que en el caso de estudiantes hombres del quintil 1 alcanzan en $6^{\circ}$ grado casi un 20\% según los padres, y cerca del $30 \%$ según estudiantes.

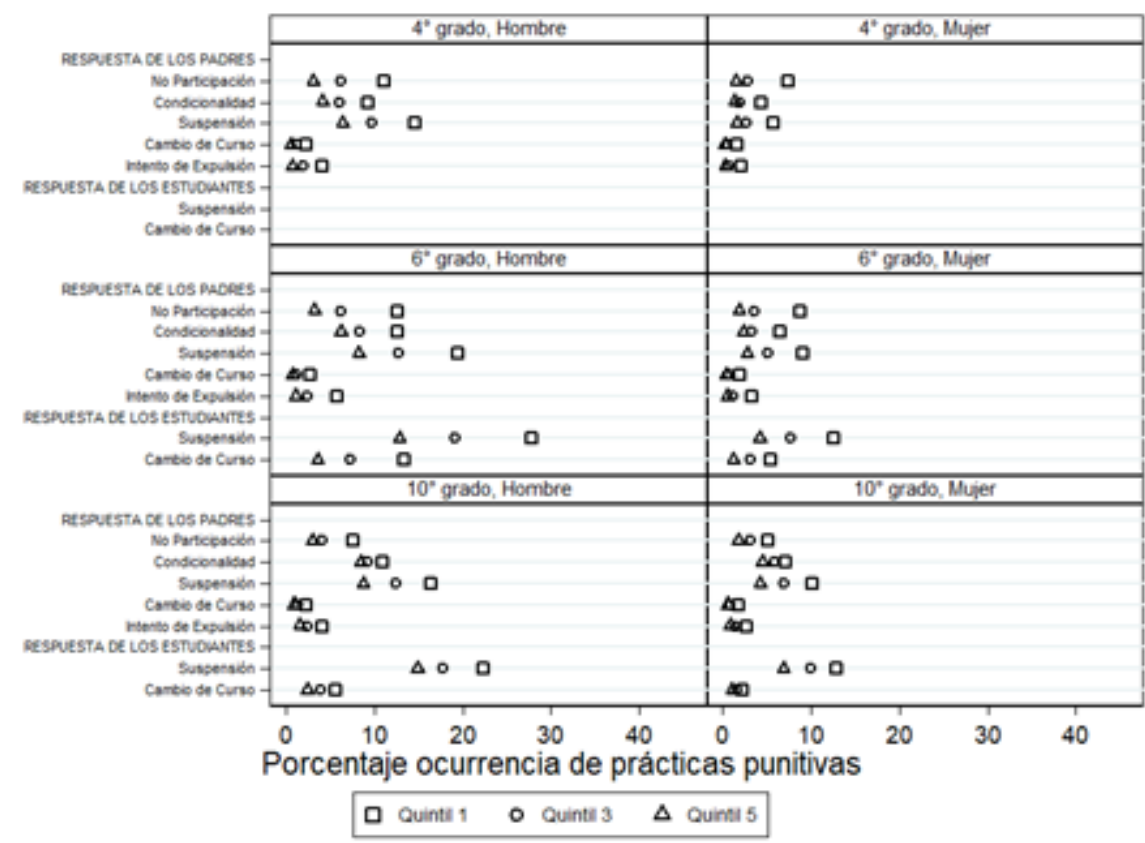

Figura 3. Porcentaje de ocurrencia informado por padres y estudiantes de aplicación de prácticas punitivas en el últimos dos años diferenciado por sexo y quintil socioeconómico Fuente: Elaboración propia.

Cuadro 3. Porcentaje percibido por padres y estudiantes de aplicación de prácticas punitivas en el último mes

\begin{tabular}{lccc}
\hline \multicolumn{1}{c}{ PráCTICA PUNITIVA } & $\mathbf{4}^{\circ}$ GRADO & $\mathbf{6}^{\circ}$ GRADO & $\mathbf{1 0}^{\circ}$ GRADO \\
\hline Percepción de Padres & & & \\
\hline Anotaciones Negativas & $23,5 \%(207.778)$ & $32,5 \%(197.766)$ & $29,3 \%(165.273)$ \\
Envío a Inspectoría & $13,6 \%(206.059)$ & $16,7 \%(196.109)$ & $12,2 \%(164.275)$ \\
\hline Percepción de Estudiantes & & & \\
\hline Anotaciones Negativas & & $50,8 \%(215.899)$ & $42,1 \%(199.077)$ \\
Envío a Inspectoría & & $25,7 \%(215.543)$ & $18,0 \%(198.657)$ \\
\hline
\end{tabular}

Nota: En paréntesis el total de observaciones para cada pregunta de los cuestionarios respectivos. Fuente: Elaboración propia.

La ocurrencia de las prácticas punitivas -anotaciones negativas y envío a inspectoría- es frecuente, y pueden ser consideradas de uso diario o común (cuadro 3). Se observa una alta 
persistencia de este tipo de prácticas, principalmente las anotaciones negativas, en todas las escuelas primarias y secundarias del país. Las diferencias en las percepciones de padres y estudiantes para este tipo de prácticas tienen diferencias extremadamente altas.

En la figura 4 observamos las diferencias para estas prácticas por sexo y nivel socioeconómico. Los resultados revelan nuevamente que la aplicación de estos mecanismos es mucho más elevada en establecimientos donde los estudiantes pertenecen a los grupos más vulnerables de la sociedad, donde a menor NSE, mayor es la frecuencia de uso. Asimismo, los estudiantes hombres son los que se ven más afectados por la aplicación de estas prácticas coercitivas por parte de las autoridades de los establecimientos.

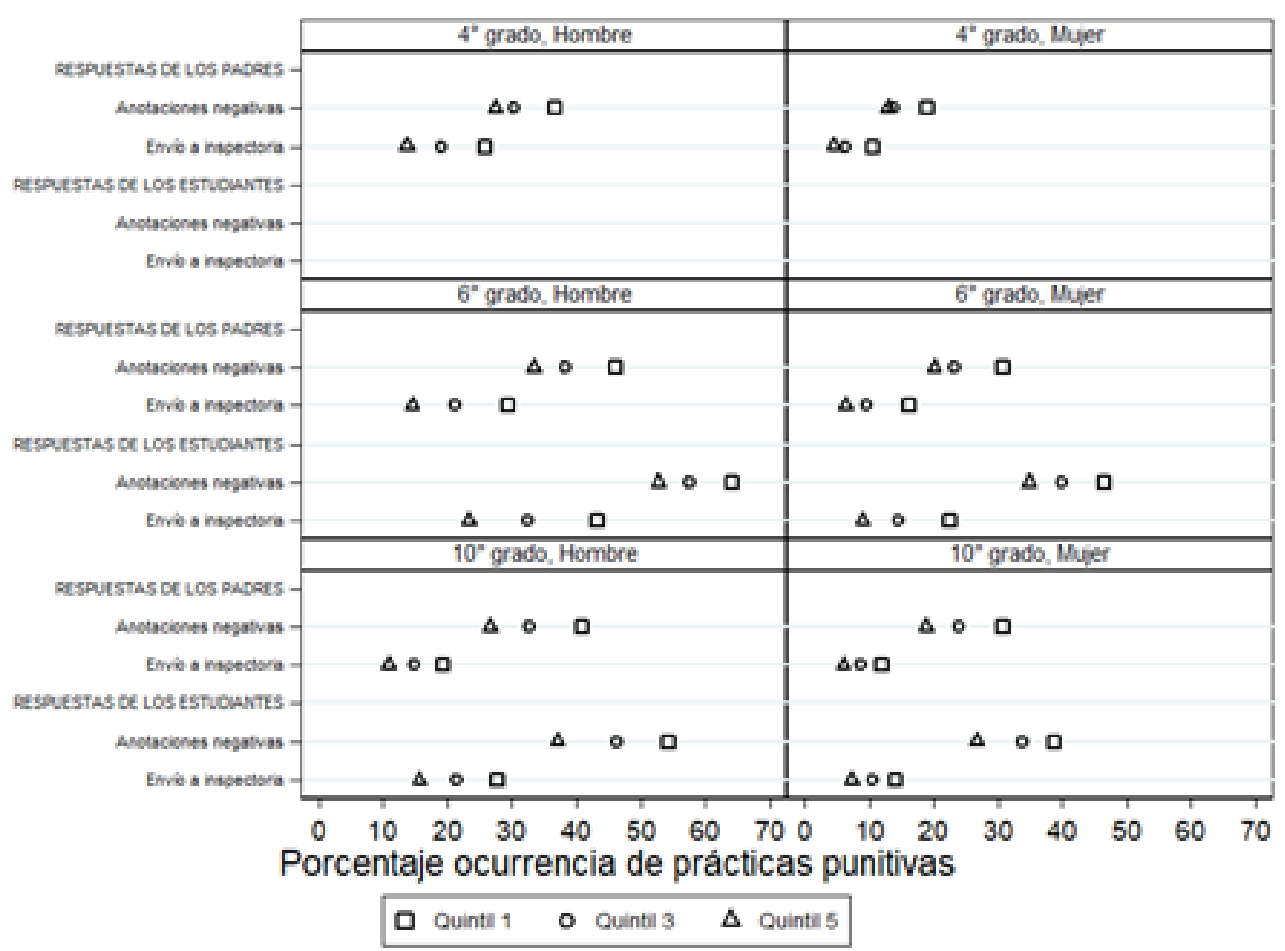

Figura 4. Porcentaje ocurrencia informado por padres y estudiantes de aplicación de prácticas punitivas durante el último mes diferenciado por sexo y quintil socioeconómico Fuente: Elaboración propia.

\subsection{Concurrencia de prácticas punitivas y de ordenamiento académico}

Para indagar en la concurrencia entre las prácticas punitivas y de ordenamiento académico se comparó solo aquellos establecimientos que en el 2018 realizaban algún tipo de agrupamiento según la estimación, dejando fuera del análisis aquellos establecimientos que realizaban agrupamiento esporádico. A diferencia de los resultados antes presentados, los resultados en esta sección se obtuvieron a nivel de establecimientos; determinando, al igual que con las prácticas de ordenamiento, cuán frecuente es la utilización de estas prácticas a nivel escolar. Para esta estimación se pesó los resultados por el número de estudiantes de cada establecimiento, buscando reconocer las diferencias asociadas al tamaño de matrícula.

Los resultados muestran que existiría una concurrencia de ambas prácticas, donde la utilización de algunas prácticas punitivas sería significativamente más frecuente en 
establecimientos que realizan prácticas de ordenamiento académico, diferencias que en los casos más extremos son solo de poco más de $2 \%$ (ver figura 5). Además, dependiendo del tipo de agrupamiento utilizado, existiría una focalización de las distintas prácticas punitivas por nivel.

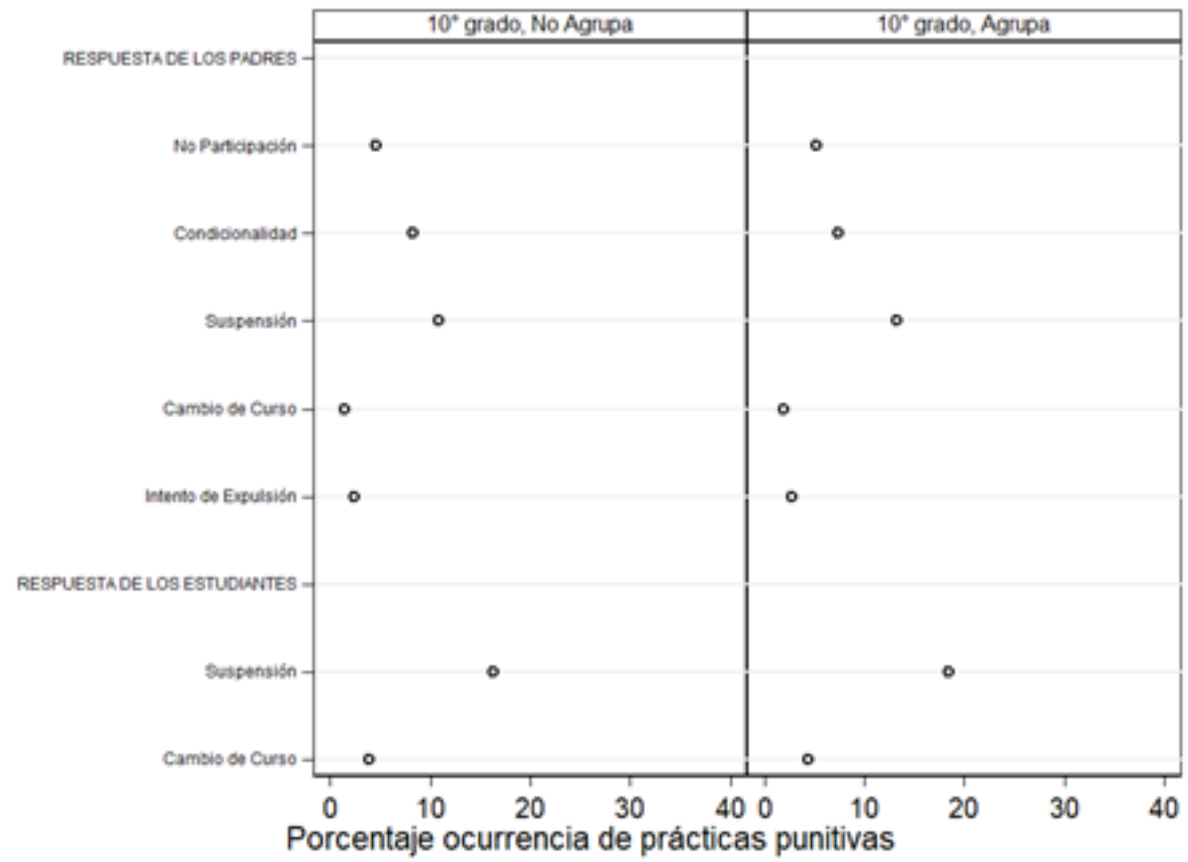

Figura 5. Porcentaje ocurrencia informado por padres y estudiantes de aplicación de prácticas punitivas durante los últimos dos años, diferenciando por si hacen agrupamiento inicial en educación secundaria Fuente: Elaboración propia.

Las principales diferencias observadas estuvieron concentradas en la educación secundaria (figura 6). En este nivel, las diferencias entre el promedio de las prácticas punitivas informadas por los padres en los colegios que ordenaban académicamente, respecto de los que no lo hacían, se presentaban en las prácticas de no participación $(0,9 \%)$, suspensión $(2,2 \%)$, cambio de curso $(0,6 \%)$ e intento de expulsión $(0,7 \%)$, mostraron ser significativamente mayores. Igual resultado se observó con las diferencias observadas en las prácticas informadas por los estudiantes respecto de cambio de curso $(1,0 \%)$ y suspensión (1,9\%).

A diferencia de los mecanismos de reordenamiento, las prácticas punitivas serían más frecuentes en educación primaria en los establecimientos que realizan agrupamiento académico (figura 7). En esta se observa que existirían diferencias significativas en la diferencia entre quienes agrupan y no agrupan en los reportes de los padres sobre condicionalidad ( $1,0 \%$ en $4^{\circ}$ grado y $1,9 \%$ en $6^{\circ}$ grado), suspensión ( $1,0 \%$ en $4^{\circ}$ grado y $1,4 \%$ en $6^{\circ}$ grado), cambio de curso ( $0,3 \%$ en $4^{\circ}$ y en $6^{\circ}$ grado) e intento de expulsión $(2,1 \%$ en $6^{\circ}$ grado). Así mismo, las diferencias en los reportes de los estudiantes, como la práctica de cambio de curso ( $0,9 \%$ en $6^{\circ}$ grado) y suspensión $\left(2,1 \%\right.$ en $6^{\circ}$ grado) también mostraron ser significativas. 


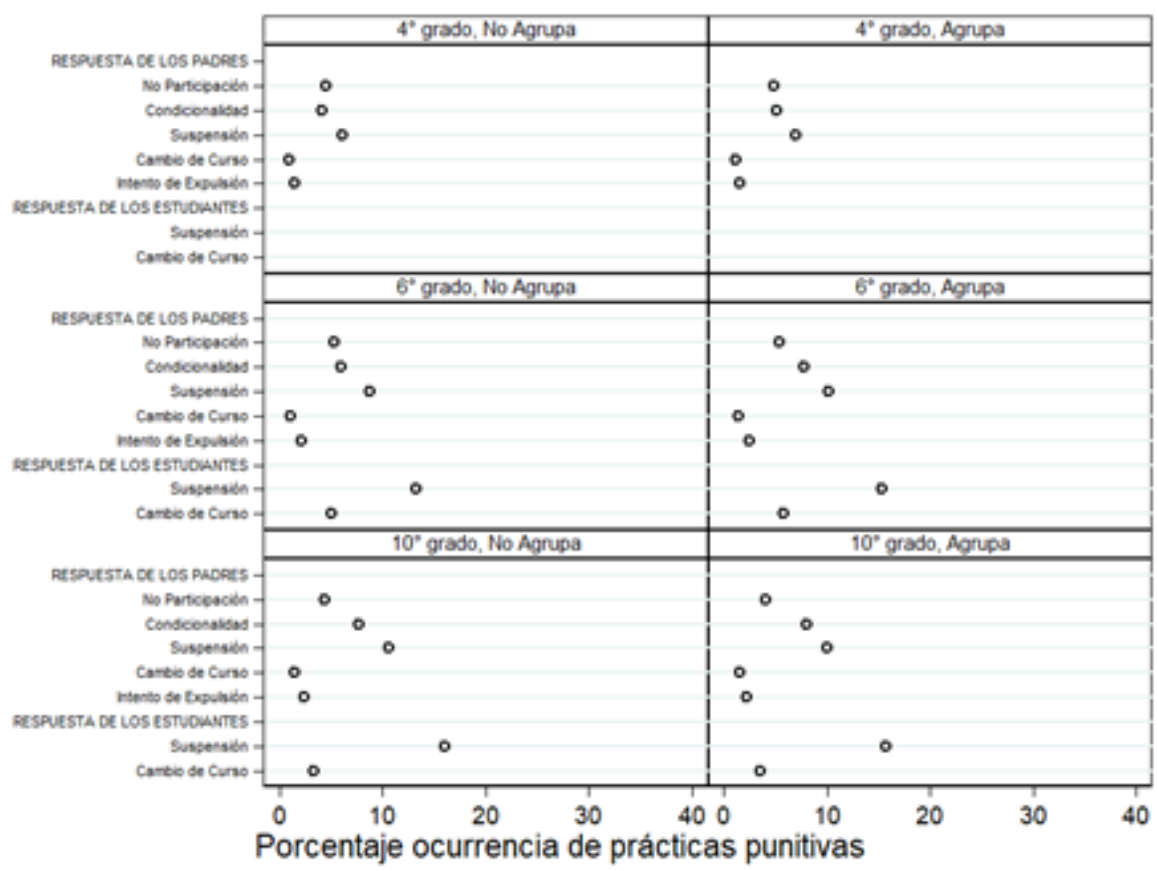

Figura 6. Porcentaje ocurrencia informado por padres y estudiantes de aplicación de prácticas punitivas durante los últimos dos años, diferenciando si hacen reordenamiento académico

Fuente: Elaboración propia.

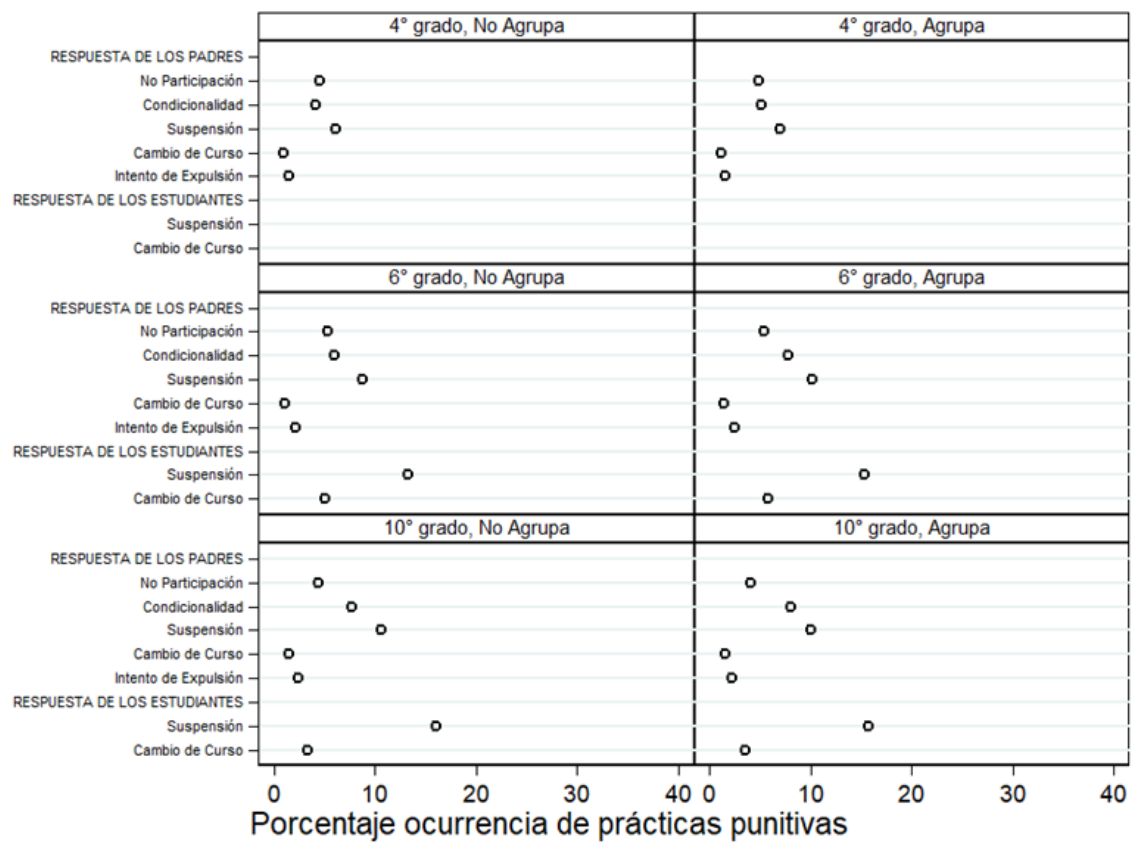

Figura 7. Porcentaje ocurrencia informado por padres y estudiantes de aplicación de prácticas punitivas durante los últimos dos años, diferenciando si hacen agrupamiento académico

Fuente: Elaboración propia. 
Finalmente, al observar los resultados diferenciando por NSE de los establecimientos, se observa nuevamente que mientras menor sea el NSE, mayor es la frecuencia de uso de prácticas punitivas, tanto entre los colegios que ordenan académicamente como entre los que no lo hacen. La figura 8, muestra los resultados obtenidos para los establecimientos que realizan agrupamiento académico. Como se puede observar, se exacerban los hallazgos descritos anteriormente, para $4^{\circ}$ y $6^{\circ}$ grado, en los establecimientos de menor NSE.

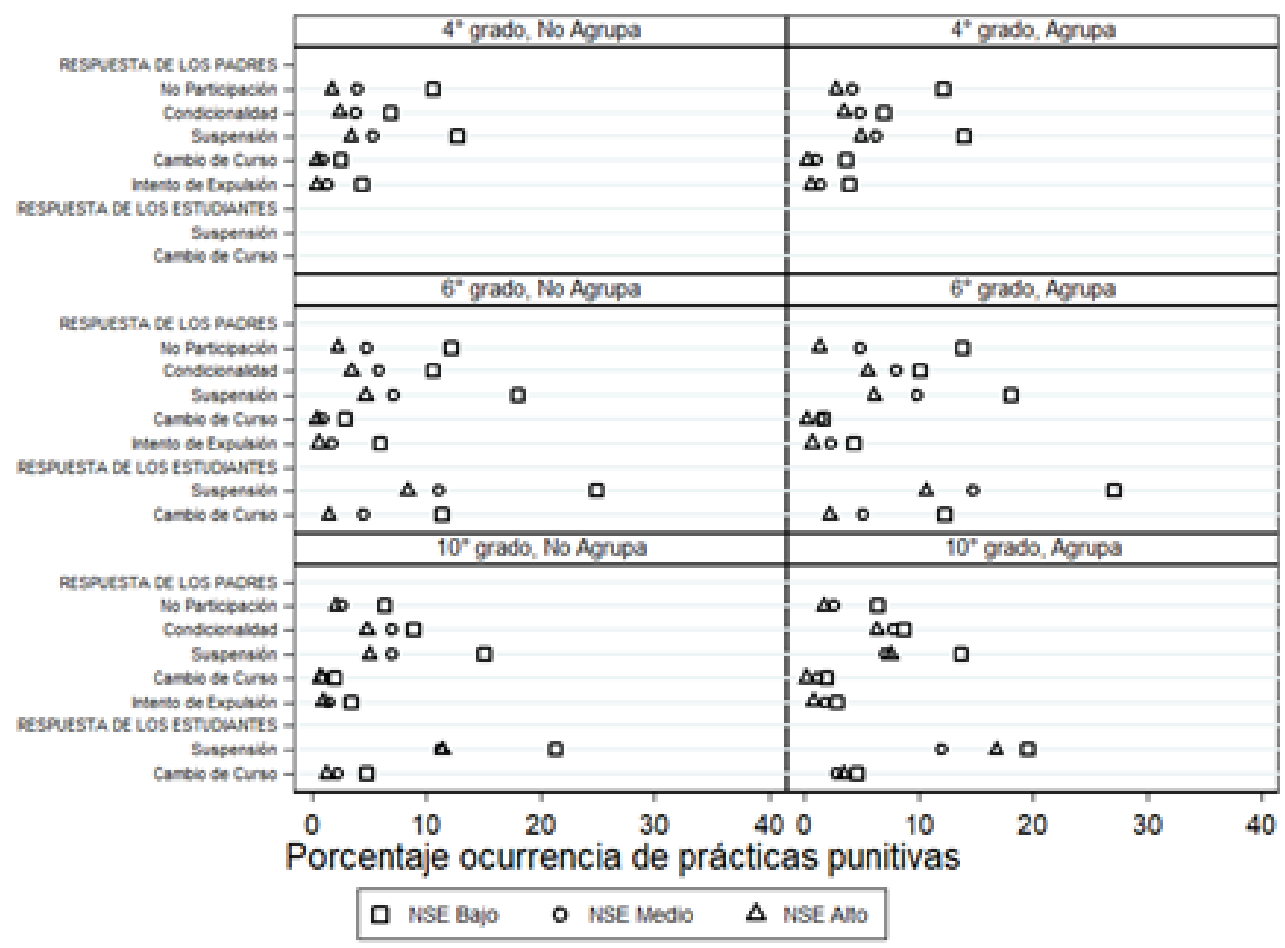

Figura 8. Porcentaje de ocurrencia informado por padres y estudiantes de aplicación de prácticas punitivas durante los últimos dos años diferenciadas por nivel socioeconómico y si realizan agrupamiento académico

Fuente: Elaboración propia.

\section{Discusiones y conclusión}

La segregación escolar no es solo un fenómeno entre escuelas, sino que ocurre y se expresa también al interior de ellas. En este estudio nos aproximamos a la descripción y análisis de formas menos visibles de segregación escolar que ocurren al separar a estudiantes con base en su rendimiento académico, así como al castigarlos por su mal comportamiento, excluyéndolos del aula y de los espacios de aprendizaje.

Los resultados muestran que ser de sexo masculino y en condición de pobreza caracterizan la ocurrencia de prácticas punitivas al interior de las escuelas chilenas. Esto implica una distribución no equitativa de los recursos escolares disponibles al servicio de cierto tipo de estudiantes por sobre otros, colocando entonces a los estudiantes con las características anteriormente descritas, en mayor riesgo de ser sujetos a prácticas de segregación y de exclusión por aplicación de este tipo de medidas. Lo que es consistente con la evidencia internacional para estas prácticas (APA, 2008; Calvin, Gurel y Barber, 2017; Camacho y Krezmien, 2019; Cobeña, 2020; Giménez et al., 2020; Murillo y Hernández, 2020; Palacios 
et al., 2019; Peguero y Bracy, 2014; Sangsurin, Chusorn y Agsonsua, 2019; SteengbergerHu, Makel y Olszewski-Kubilius, 2016).

Estos hallazgos muestran cómo el fenómeno de la segregación educativa aparece como una micropráctica o un tipo de segregación (Bellei, 2013) que se reproduce en la cotidianidad escolar (Cobeña, 2020). Se trataría de patrones naturalizados de comportamiento profesional regidos con base en ciertas distinciones que los directivos y profesores hacen de sus estudiantes con base al rendimiento académico y su conducta en el aula. Esto podría ser entendido como un antecedente más de la hipersegregación del sistema educativo chileno (González, 2017), tratándose de un mecanismo de intensificación de las condiciones de exclusión social para algunos estudiantes, que no sólo asisten a escuelas altamente segregadas, sino que experimentarían una serie de prácticas que los excluiría de la actividad pedagógica formal, mediante acciones de disciplina desproporcionada y ordenamiento según habilidades académicas (Marcucci y Emesky, 2020; Ramberg, 2016)

En el caso de las prácticas de segregación académica intraescolar e interescolar, un resultado inesperado del análisis descriptivo efectuado sobre una ventana de tiempo de doce años fue la disminución sistemática de estas prácticas desde el año 2014 en adelante. Los resultados indican que durante el 2014-2015, aumentó progresivamente el número de escuelas que no realiza este tipo de prácticas. A su vez, en este mismo período, comenzó a disminuir progresivamente el número de escuelas que realiza agrupamiento y reordenamiento académico, especialmente notorio en aquellos centros escolares que solo tienen enseñanza secundaria. Es notorio, no sólo por la disminución de instituciones que la realizan, considerando su uso común, sino porque en Chile ni la agrupación inicial ni el reordenamiento académico están prohibidos ni siquiera regulados por ley (Treviño et al., 2018), pudiendo hipotetizar el efecto performativo de otras políticas asociadas a inclusión escolar, y que comienzan a ponerse en la palestra del escenario nacional e internacional en las últimas décadas (Manghi et al., 2020).

Durante este periodo, en Chile se discutía el sentido general y las justificaciones éticas y teóricas para la promulgación de legislación de garantía de derechos para el acceso escolar y de diversificación de la composición social de los estudiantes en las escuelas financiada por el Estado, mediante un único sistema de postulación escolar (Ley de Inclusión -Ley $\mathrm{N}^{\circ}$ 21.015-, 2015). Y si bien, el alcance de esta investigación no buscó evaluar esta legislación en los años de promulgación e implementación, es interesante explorar en futuros estudios el efecto performativo de este dispositivo (Carrasco, 2013; Sisto, 2019) en la construcción de nuevos mecanismos para la gestión de la heterogeneidad estudiantil respecto a la distribución intra-escuela de los estudiantes en función de su rendimiento académico, y que sostienen el argumento sobre la disminución de este prácticas menos visibles.

En cuanto a las prácticas de disciplina punitiva, no fue posible describir su evolución en el tiempo, pues a la fecha solo se cuenta con resultados para una cohorte de estudiantes. No obstante, estos hallazgos sugieren que algunas de estas prácticas tienen una frecuencia mayor que en otros países. Por ejemplo, los padres chilenos informan que cerca de un $2 \%$ de los estudiantes sufrió algún intento de expulsión escolar durante el último año. Si bien esta cifra no corresponde a expulsiones efectivas, es muy probable que un alto porcentaje sí haya sido efectiva; comparando con la realidad norteamericana, que informó una tasa de estudiantes expulsados de 0,22\% (Digest of Education Statistics, 2018). De manera 
similar, el $15,1 \%$ de estudiantes chilenos de $6^{\circ}$ grado, y el $15,7 \%$ de $10^{\circ}$ grado informaron que los habían suspendido el año anterior. Esta cifra es mayor al 7,3\% (para hombres) y 3,2\% (para mujeres) reportadas en Estados Unidos (Digest of Education Statistics, 2018), porcentajes bastante distantes de los cerca de $12,4 \%$ de hombres y $5,5 \%$ de mujeres que se les habría aplicado alguna suspensión en Chile.

Una posible explicación a este escenario, requiere entender el contexto del uso de las anotaciones negativas y envíos a inspectoría. Estas suelen ser prácticas de uso diario y coercitivas que tienen a disposición tanto profesores como inspectores para mantener la disciplina al interior de las aulas (Camacho y Krezmien, 2019; Marcucci y Elmesky, 2020) y también fuera de ellas (Umeh, Bumpus y Harris, 2019). Asimismo, la información de estas no suele ser entregada a los padres ni informadas regularmente a los propios estudiantes (López et al., 2019), lo que sin duda genera un proceso de distanciamiento entre la escuela, las familias y las posibilidades educativas que surgen en dicha relación (Bell, 2020; Murillo y Hernández, 2020).

Los análisis preliminares de la concurrencia de prácticas de ordenamiento académico con diversas prácticas punitivas indican que éstas últimas son más frecuentes entre los colegios que ordenan académicamente, diferencias que en la mayor parte de los casos son estadísticamente significativas. No obstante, su aplicación es relativamente independiente al de las prácticas de ordenamiento académico observadas en Chile; lo que desafía al país a avanzar en políticas, programas y estrategias específicas para modificar las prácticas y las culturas al interior de los establecimientos educativos chilenos, por otras de reparación y diálogo en la resolución de los conflictos disciplinarios (Augustine et al., 2018; Marcucci, 2019; Weaver y Swank, 2020). La consigna de una escuela más inclusiva implica entonces, considerar aquellas prácticas menos visibles de segregación y exclusión escolar que informan los estudiantes y padres como frecuentes, y que desde estos hallazgos están sobre-representadas en aquellas poblaciones justamente más vulnerables (Lehmann et al., 2020; Losen, 2011; Weaver y Swank, 2020).

Entre las limitaciones de este estudio, consignamos que la metodología para identificar agrupamiento/ordenamiento académico entre aulas solo es aplicable a los establecimientos que tienen dos o más cursos, lo que deja fuera muchos mecanismos utilizados y descritos en la literatura de ordenamiento al interior de las aulas, como puede ser la creación de grupos y ordenar las salas de clase de acuerdo al rendimiento y comportamiento de los alumnos, entre otros. Por otra parte, nuestro acercamiento a las prácticas punitivas consiste en lo que informan los estudiantes y sus padres. Al no existir un registro público de esto, constituyen la mejor aproximación posible al fenómeno en estudio.

A futuro, sugerimos el estudio de la evolución del uso de las prácticas punitivas en escuelas chilenas, así como estudios comparados en los países de habla hispana. Así también, es necesario estudiar si y cuál es el nivel de incidencia de las prácticas punitivas y de segregación por grupo curso en indicadores claves educativos, como es el desempeño académico, la repitencia y rezago educativo, y la deserción. Desde una perspectiva cualitativa, es necesario comprender cómo se configuran discursivamente estas prácticas en el imaginario social y cómo se despliegan en escenarios concretos. Además, sugerimos indagaciones cualitativas de corte crítico que exploren las condiciones de posibilidad sobre las que se sustentan y sostienen en el tiempo estas prácticas de segregación menos visibles. Posiblemente la persistencia de los paradigmas conductistas y la valoración positiva del 
castigo como mecanismo formativo presentes aún en las comunidades escolares, estén a la base de su sustentabilidad.

\section{Agradecimientos}

Esta investigación fue posible gracias al Proyecto FONDECYT REGULAR 1191267; al Proyecto FONIDE 1900118; al Programa de Investigación Asociativa CIE-160009 de la Agencia Nacional de Investigación y Desarrollo de Chile (ANID, Centro de Investigación para la Educación Inclusiva); y al Fondo Basal FB-0003 del Centro de Investigación Avanzada en Educación, Universidad de Chile. Las preguntas referidas a prácticas punitivas fueron incorporadas en los Cuestionarios de Calidad y Contexto de la Educación 2018 de la Agencia de Calidad de la Educación, luego de haber sido seleccionada la propuesta en la convocatoria para formular propuestas de preguntas en los mismos.

\section{Referencias}

Allende, C., Valenzuela, J. P. y Diaz, R. (2018). School segregation in Chile. En A. Farazmand (Ed.), Global encyclopedia of public administration, public policy, and governance. Springer International Publishing. https://doi.org/10.1007/978-3-319-31816-5

APA. (2008). Are zero tolerance policies effective in the schools? An evidentiary review and recommendations. American Psychologist, 63, 852-862. https://doi.org/10.1037/0003066X.63.9.852

Augustine, C., Engberg, J., Grimm, E., Lee, E., Wang, K., Christianson, A. y Joseph, A. (2018). Can restorative practices improve school climate and curb suspensions? An evaluation of the impact of restorative practices in a mid-sized urban school district. Rand Corporation, OAES.

Ascorra, P., Carrasco, C., López, V. y Morales, M. (2019). Políticas de convivencia escolar en tiempos de rendición de cuentas. Archivos Analíticos de Politicas Educativas, 27(31). https://doi.org/10.14507/epaa.27.3526

Ascorra, P., López, V., Carrasco, C., Pizarro, I., Cuadros, O. y Núñez, C. (2018). Significados atribuidos a la convivencia escolar por equipos directivos, docentes y otros profesionales de escuelas chilenas. Psykhe, 27(1), 1-12. http://doi.org/10.7764/psykhe.27.1.1214

Belfi, B., Goos, M., De Fraine, B. y Van Damme, J. (2012). The effect of class composition by gender and ability on secondary school students' school well-being and academic self-concept: A literature review. Educational Research Review, 7(1), 62-74. https://doi.org/10.1016/j.edurev.2011.09.002

Bell, C. (2020). Maybe if they let us tell the story I wouldn't have gotten suspended: Understanding black students' and parents' perceptions of school discipline. Children and Youth Services Revierw, $110(2)$. https://doi.org/10.1016/j.childyouth.2020.104757

Bellei, C. (2009). The private-public school controversy: The case of Chile. En P. Peterson y R. Chakrabarti (Eds.), School choice international (pp. 165-192). MIT Press.

Bellei, C. (2015). El gran experimento: Mercado y privatización de la educación chilena. LOM ediciones.

Betts, J. y Shkolnik, J. (2000). The effects of ability grouping on student achievement and resource allocation in secondary schools. Economics of Education Revierw, 19(1), 1-15. https://doi.org/10.1016/SO272-7757(98)00044-2

Bolick, K. y Rogowsky, B. (2016). Ability grouping is on the rise, but should it be? Journal of Education and Human Development, 5(2), 4-45. https://doi.org/10.15640/jehd.v5n2a6 
Calvin, G., Gurel, S. y Barber, B. (2017). State-level analysis of school punitive discipline practices in Florida. Behavioral Disorder, 42(2), 65-80. https://doi.org/10.1177/0198742916688652

Campoy, I. (Dir.). (2019). Educación inclusiva y medidas sancionadoras en el ámbito educativo: La situación de niños y niñas con trastornos de apego. Instituto de Derechos Humanos Bartolomé de las Casas, Universidad Carlos III de Madrid.

Camacho, K. y Krezmien, M. (2019). A statewide analysis of school discipline policies and suspension practices. Preventing School Failure: Alternative Education for Children and Touth, 64(1), 55-66. https://doi.org/10.1080/1045988X.2019.1678010

Carrasco, A. (2013). Mecanismos performativos de la institucionalidad educativa en Chile: Pasos hacia un nuevo sujeto cultural. Observatorio Cultural, 15, 4-10.

Ceardi, A., López, V. y Valdez. R. (abril, 2018) Disciplinary punitive practices and its relation to Chilean students' perception of school climate practices. Ponencia presentada en American Educational Research Association Annual Meeting, Nueva York.

Chmielewski, A. (2014). An international comparison of achievement inequality in within-and between-school tracking systems. American Journal of Education, 120(3), 293-324. https://doi.org/10.3102/0002831213489843

Chmielewski, A., Dumont, H. y Trautwein, U. (2013). Tracking effects depend on tracking type: An international comparison of students' mathematics self-concept. American Educational Research Journal, 5o(5), 925-957. https://doi.org/10.3102/0002831213489843

Cobeña, P. (2020). Exclusión educativa de personas con discapacidad: Un problema pedagógico. REICE. Revista Iberoamericana sobre Calidad, Eficacia y Cambio en Educación, 18(1), 65-81. https://doi.org/10.15366/reice2020.18.1.004

Contreras, D., Brante, M., Espinoza, S. y Zuñiga, I. (2020). The effect of the integration of students with special educational needs: Evidence from Chile. International Journal of Educational Development, 74, 102-163. https:// doi.org/10.1016/j.ijedudev.2020.102163

Digest of Education Statistics. (2018). The condition of education 2018. NCES 2018-144. National Center for Education Statistics.

Duflo, E., Dupas, P. y Kremer, M. (2011). Peer effects, teacher incentives, and the impact of tracking: Evidence from a randomized evaluation in Kenya. The American Economic Review, 101(5), 1739-1774. https://doi.org/10.1257/aer.101.5.1739

Dupriez, V. (2010). Methods of grouping learners at school. International Institute for Educational Planning.

Dustmann, C., Puhani, P. A. y Schönberg, U. (2017). The long-term effects of early track choice. The Economic Journal, 127(603), 1348-1380. https://doi.org/10.1111/ecoj.12419

Duk, C. y Murillo, F. J. (2019). Segregación escolar y meritocracia. Revista Latinoamericana de Educación Inclusiva, 13(1), 11-13. https://doi.org/10.4067/So718-73782019000100011

Elacqua, G. y Santos, H. (2013). Los efectos de la elección escolar en la segregación socioeconómica en Chile: Un análisis georreferenciado. Espacio Público.

Figlio, D. y Page, M. (2002). School choice and the distributional effects of ability tracking: Does separation increase inequality? Journal of Urban Economics, 51(3), 497-514. https://doi.org/10.1006/juec.2001.2255

Francis, B., Hodgen, J., Craig, N., Taylor, B., Archer, L., Mazenod, A., Tereshchenko, A. y Connolly, P. (2019). Teacher quality and attainment grouping: The role of within-school teacher deployment in social and educational inequality. Teaching and Teacher Education, 77(1), 183-192. https://doi.org/10.1016/j.tate.2018.10.001 
Francis, B., Connelly, P., Archer, L., Hodgen, J., Mazenod, A., Pepper, D., Sloan, S., Taylor, B., Tereshchenko, A. y Travers, M. C. (2017). Attainment grouping as self-fulfilling prophesy? A mixed methods exploration of self-confidence and set level among year 7 students. International Journal of Educational Research, 86, 96-108. https://doi.org/10.1016/j.ijer.2017.09.001

Giménez, V., Thieme, C., Prior, D. y Tortosa-Ausina, E. (2020). Evaluation and determinants of preschool effectiveness in Chile.

www.doctreballeco.uji.es/wpficheros/Gimenez_et_al_02_2020.pdf

González, R. (2017). Segregación educativa en el sistema chileno desde una perspectiva comparada. MINEDUC.

Gregory, A., Clawson, K., Davis, A. y Gerewitz, J. (2016). The promise of restorative practices to transform teacher-student relationships and achieve equity in school discipline. Journal of Educational and Psychological Consultation, 26(4), 325-353. https://doi.org/10.1080/10474412.2014.929950

Ireson, J. y Hallam, S. (2001). Ability grouping in education. SAGE.

López, V., Litichever, L., Valdés, R. y Ceardi, A. (2019). Traduciendo políticas de convivencia escolar: Análisis de dispositivos sociotécnicos en Argentina y Chile. Psicoperspectivas, 18(1), 1-15. https://doi.org/10.5027/psicoperspectivas-vol18-issue1-fulltext-1484

López, V., Carrasco, C., Morales, M., Ayala, A., López, J. y Karmy, M. (2011). Individualizando la violencia escolar: Análisis de prácticas discursivas en una escuela municipal de la región de Valparaíso. Psykhe, 20(2), 17-33. https://doi.org/10.4067/So718-22282011000200002

Losen, D. (2011). Discipline policies, successful schools, and racial justice. National Education Policy Center.

Lehmann, P., Chouhy, C., Singer, A., Stevens, J. y Gertz, M. (2020). Group threat, racial/ethnic animus, and punitiveness in Latin America: A multilevel analysis. Race and Justice. https://doi.org/10.1177/2153368720920347

Magendzo, A., Toledo, M. y Gutiérrez, V. (2012). Descripción y análisis de la ley sobre violencia escolar (No. 20.536): Dos paradigmas antagónicos. Estudios Pedagógicos, 39(1), 377-391. https://doi.org/10.4067/s07 18-07052013000100022

Manghi, D., Solar, Conejeros, M. L. Bustos, A., Aranda, I., Vega, V. y Díaz, K. (2020). Comprender la educación inclusiva chilena: Panorama de políticas e investigación educativa. Cadernos de Pesquisa, 50(175), 114-134. https://doi.org/10.1590/198053146605

Marcucci, O. (2019). Implicit bias in the era of social desirability: Understanding antiblackness in rehabilitative and punitive school discipline. Urban Review, 52, 47-71. https://doi.org/10.1007/s1 1256-019-00512-7

Marcucci, O. y Elmesky, R. (2020). Advancing culturally relevant discipline: An ethnographic microanalysis of disciplinary interactions with black students. Urban Education. https://doi.org/10.1177/0042085920909165

Mazenod, A., Francis, B., Archer, L., Hodgen, J., Taylor, B., Tereshchenko, A. y Pepper, D. (2018). Nurturing learning or encouraging dependency? Teacher constructions of students in lower attainment groups in English secondary schools. Cambridge Journal of Education, 49(1), 53-68. https://doi.org/10.1080/0305764X.2018.1441372

Morales, M. y López, V. (2019). Políticas de convivencia escolar en América Latina: Cuatro perspectivas de comprensión y acción. Education Policy Analysis Archives, 5, 1-25. https://doi.org/10.14507/epaa.27.3800 
Muñoz, M., Lucero, B., Cornejo, C., Muñoz, P. y Araya, N. (2014). Convivencia y clima escolar en una comunidad educativa inclusiva de la Provincia de Talca, Chile. Revista Electrónica de Investigación Educativa, 16(2), 16-32.

Murillo, F. J. y Hernández, R. (2020). Does parental involvement matter in children's performance? A Latin American primary school study. Revista de Psicodidáctica, 25(1), 13-22. https://doi.org/10.1016/j.psicod.2019.10.002

Oakes, J. (2008). Keeping track: Structuring equality and inequality in an era of accountability. Teachers College Record, 110, 700-712.

OCDE. (2013). PISA 2012 results: What makes schools successful? Resources, policies and practices. OECD Publishing.

OCDE. (2016). PISA 2015 results. Policies and practices for successful schools. OECD Publishing.

Palacios, D., Dijkstra, J. K., Villalobos, C., Treviño, E., Berger, C., Huisman, M. y Veenstra, R. (2019). Classroom ability composition and the role of academic performance and school misconduct in the formation of academic and friendship networks. Journal of School Psychology, 74, 58-73. https://doi.org/10.1016/j.jsp.2019.05.006

Parcerisa, L. y Falabella, A. (2017). La consolidación del estado evaluador a través de políticas de rendición de cuentas: Trayectorias, producción y tensiones en el sistema educativo. Archivos Analíticos de Políticas Educativas, 25(89). https://doi.org/10.14507/epaa.25.3177

Peguero, A. y Bracy, N. (2014). School order, justice, and education: Climate, discipline practices, and dropping out. Journal of Research on Adolescence, 25(3), 412-426.

https://doi.org/10.1111/jora.12138

Ramberg, J. (2016). The extent of ability grouping in Swedish upper secondary schools: A national survey. International Journal of Inclusive Education, 20(7), 685-710. http://doi.org/10.1080/13603116.2014.929187

Richard, J., Schneider, B. y Mallet, P. (2012). Revisiting the whole-school approach to bullying: Really looking at the whole school. School Psychology International, 33(3), 263-284. https://doi.org/10.1177/0143034311415906

Sangsurin, K., Chusorn, P. y Agsonsua, P (2020). A model of causal relationships affecting the effectiveness of primary schools under Khon Kaen primary education service area. International Journal of Higher Education, 9(1), 230-236.

https://doi.org/10.5430/ijhe.v9n1p230

Sisto, V. (2019). Managerialismo versus prácticas locales: La decolonización del discurso managerial desde la vida de la escuela. Cuadernos de Administración, 32(58).

https://doi.org/10.11144/Javeriana.cao.32-58.mvpl

Skiba, R. y Knesting, K. (2001). Zero tolerance, zero evidence: An analysis of school disciplinary practice. New Directions for Touth Development, 92, 17-43. https://doi.org/10.1002/yd.23320019204

Skiba, R. y Rausch, M. (2013). Zero tolerance, suspension, and expulsion: Questions of equity and effectiveness. En C. Evertson y C. Weinstein (Eds.), Handbook of classroom management: Research, practice, and contemporary issues (pp. 1073-1100). Routledge.

Skiba, R. Arredondo, M. y Williams, N. (2014). More than a metaphor: The contribution of exclusionary discipline to a school-to-prison pipeline. Equity y Excellence in Education, 47(4), 546-564. https://doi.org/10.1080/10665684.2014.958965

Sugai, G., Sprague, J., Horner, R. y Walker, H. (2000). Preventing school violence: The use of office discipline referrals to assess and monitor school-wide discipline interventions. Journal of 
Emotional and Behavioral Disorders, 8(2), 94-101. https://doi.org/10.1177/106342660000800205

Steengberger-Hu, S., Makel, M. C. y Olszewski-Kubilius, P. (2016). What one hundred years of research says about the effects of ability grouping and acceleration on K-12 students' academic achievement: Findings of two second-order meta-analyses. Review of Educational Research, 86(4), 849-899. https://doi.org/849-899. 10.3102/0034654316675417

Tereshchenko, A., Francis, B., Archer, L., Hodgen, J., Mazenod, A., Taylor, B., Pepper, D. y Travers, M. C. (2018). Learners' attitudes to mixed attainment grouping: Examining the views of students of high, middle and low attainment. Research Papers in Education, 34(4), 425-444. https://doi.org/10.1080/02671522.2018.1452962

Trautmann M. A. (2008). Maltrato entre pares o "bullying": Una visión actual. Revista Chilena de Pediatría, 79(1), 13-20. https://doi.org/10.4067/S0370-41062008000100002

Treviño, E., Valenzuela, J. P. y Villalobos, C. (2016). Within-school segregation in the Chilean school system: What factors explain it? How efficient is this practice for fostering student achievement and equity? Learning and Individual Differences, 51, 367-375.

https://doi.org/10.1016/j.lindif.2016.08.021

Treviño, E., Wyman, I., Villalobos, C. y Romo, F. (noviembre, 2019). Reference group effect on students' socio-emotional development: Testing the incidence of ability grouping and quality of classroom interactions. Comunicación presentada en Congreso CIIE 2019. Shanghái, China

Treviño, E., Valenzuela, J. P., Villalobos, C., Béjares, C., Wyman, I. y Allende, C. (2018). Agrupamiento por habilidad académica en el sistema escolar. Nueva evidencia para comprender las desigualdades del sistema educativo chileno. Revista Mexicana de Investigación Educativa, 23(76), 45-71.

Umeh, Z., Bumpus, J. y Harris, J. (2019). The impact of suspension on participation in school-based extracurricular activities and out-of-school community service. Social Science Research, 85(2), 23-54. https://doi.org/10.1016/j.ssresearch.2019.102354

Valdés, R., López, V. y Chaparro, A. (2018). Convivencia escolar: Adaptación y validación de instrumento mexicano en Chile. Revista de Investigación Educativa, 20(3), 80-91. https://doi.org/10.24320/redie.2018.20.3.1720

Valenzuela, J. P., Bellei, C. y De Los Ríos, D. (2014). Socioeconomic school segregation in a marketoriented educational system: The case of Chile. Journal of Education Policy, 29(2), 217-241. https://doi.org/10.1080/02680939.2013.806995

Valenzuela, J. P., Villarroel, G. y Villalobos, C. (2013). Ley de subvención escolar preferencial (SEP): Algunos resultados preliminares de su implementación, Pensamiento Educativo, 5o(2), 113-131.

Veiga-Neto, A. y Corcini, M. (2012). A inclusão como dominação do outro pelo mesmo. Pedagogía y Saberes, 36(2), 57-68.

Weaver, J. y Swank, J. (2020). A case study of the implementation of restorative justice in a middle school. Research in Middle Level Education, 43(4), 1-9. https://doi.org/10.1080/19404476.2020.1733912

Williams, J., Lewis, W. Starker, T., Butler, B. y Hoon, J. (2020) The discipline gatekeeper: Assistant principals' experiences with managing school discipline in urban middle schools. Urban Education, 75(1), 23-36. https://doi.org/10.1177/0042085920908913 


\section{Breve CV de los autores}

\section{Verónica López}

Psicóloga, Pontificia Universidad Católica de Chile. Doctora en Psicología, Universidad Autónoma de Madrid, España. Profesora titular de la Escuela de Psicología de la Pontificia Universidad Católica de Valparaíso. Directora del Programa de Apoyo a la Convivencia Escolar (www.paces.cl), y Directora del Centro de Investigación para la Educación Inclusiva de la Pontificia Universidad Católica de Valparaíso (www.eduinclusiva.cl). Su investigación se centra en las condiciones que favorecen el aprendizaje escolar y el desarrollo socioemocional de niños y adolescentes, asociado a temáticas de convivencia escolar, bienestar educativo e inclusión. En la actualidad investiga sobre temáticas de exclusión escolar, disciplina y prácticas de castigo punitivo en la escuela, asociado al proyecto Fondecyt Regular 1191267 "La inclusión excluyente: prácticas de exclusión y ordenamiento en escuelas chilenas". ORCID ID: https://orcid.org/0000-0001-74053859. Email: veronica.lopez@pucv.cl

\section{Sebastián Ortiz}

Psicólogo Pontificia Universidad Católica de Valparaíso. Magíster en Psicología Educacional de la Universidad Chile. Académico del Departamento Disciplinar de Psicología de la Universidad de Playa Ancha, Valparaíso. Profesional de Investigación de la Línea 1 en Convivencia Escolar y Bienestar del Centro de Investigación del Centro de Investigación para la Educación Inclusiva (www.eduinclusiva.cl). Su interés científico ha estado centrado en las condiciones psicosociales y pedagógicas que favorecen una escuela democrática, temáticas asociadas a la violencia escolar y a las organizaciones sindicales del profesorado. ORCID ID: https://orcid.org/O000-0001-7877-3319. Email: sebastian.ortiz@pucv.cl

\section{Claudio Allende}

Ingeniero Comercial con mención en Economía y Magíster en Políticas Públicas en la Universidad de Chile; Diplomado en Políticas Públicas de la Universidad de Chicago. Investigador asociado al Centro de Investigación Avanzada en Educación de la Universidad de Chile. Ha desarrollado investigaciones en temas de educación superior, escolaridad y evaluación de políticas públicas educacionales. ORCID ID: https://orcid.org/0000-0002-6628-1787. Email: claudio.allende.gonzalez@gmail.com

\section{Juan Pablo Valenzuela}

Ingeniero Comercial, Universidad de Chile. Doctor en Economía, Universidad de Michigan-Ann Arbor, Estados Unidos. Investigador asociado al Centro de Investigación Avanzada en Educación y Académico del Departamento de Economía de la Universidad de Chile. Sus principales áreas de investigación son la economía de la educación y la inequidad social, abordando temáticas vinculadas a la segregación social y educativa, el mejoramiento escolar y la efectividad escolar en establecimiento educacionales. ORCID ID: https://orcid.org/0000-0003-0445-968X. Email: jp.valenzuelab@gmail.com 


\section{Luis González}

Ingeniero Comercial, Universidad de Chile. Profesional de Investigación del Centro de Investigación del Centro de Investigación para la Educación Inclusiva (www.eduinclusiva.cl). Ha desarrollado investigaciones en temas de educación escolar, deserción escolar y clima escolar. ORCID ID: https://orcid.org/0000-0003-2651-3528. Email: lgonzalezh@fen.uchile 